Published in The Lancet, 2017, Volume 390, No. 10093, p500-509

http://www.thelancet.com/journals/lancet/article/PIIS0140-6736(17)30572-X/fulltext

\title{
Evolutionary public health: introducing the concept
}

Jonathan CK Wells PhD [1]

Randolph M Nesse MD [2]

Rebecca Sear PhD [3]

Rufus A Johnstone PhD [4]

Stephen C Stearns PhD [5]

1. Childhood Nutrition Research Centre, UCL Great Ormond Street Institute of Child Health, London, UK

2. Centre for Evolution and Medicine, Arizona State University, Phoenix, AZ, US

3. London School of Hygiene and Tropical Medicine, London, UK

4. Department of Zoology, University of Cambridge, Cambridge, UK

5. Department of Ecology and Evolutionary Biology, Yale University, New Haven, CT, US

Corresponding author:

Jonathan Wells

Childhood Nutrition Research Centre

UCL Great Ormond Street Institute of Child Health

30 Guilford Street

London, UK

jonathan.wells@ucl.ac.uk 


\section{Abstract}

The emerging discipline of evolutionary medicine is breaking new ground in understanding why people get sick. However, the value of evolutionary analyses of human physiology and behaviour is only beginning to be recognized in the field of public health. Core principles come from life history theory, which analyses the allocation of finite amounts of energy among four competing functions - maintenance, growth, reproduction and defence. A central tenet of evolutionary theory is that organisms are selected to allocate energy and time to maximize reproductive success, rather than health or longevity. Ecological interactions that influence mortality risk, nutrient availability and pathogen burden shape energy-allocation strategies throughout the life-course, thereby impacting diverse health outcomes. Public health interventions could improve their efficacy by incorporating an evolutionary perspective. In particular, evolutionary approaches offer new opportunities to address the complex challenges of global health, where populations are differentially exposed to the metabolic consequences of poverty, high fertility, infectious diseases, and rapid changes in nutrition and lifestyle. The impact of specific interventions may depend on broader factors shaping life expectancy. Amongst the important tools in this approach are mathematical models, which can explore likely benefits and limitations of interventions in silico, prior to their implementation in human populations. 


\section{Introduction}

Public health aims to prevent disease, promote health and prolong life among human populations through the organized efforts of society. ${ }^{1}$ It is intuitive that improving living conditions should benefit health, but from an evolutionary perspective this assumption is simplistic. Natural selection has not shaped organisms for maximum health, but rather to maximize their reproductive success ('genetic fitness', see supplementary online glossary). Consequently, public health interventions may not always achieve exactly what they intended.

Consider an example from rural Ethiopia, where a water development scheme aimed to decrease the daily energy burden on women who carried water up to $30 \mathrm{~km}$ in clay pots. ${ }^{2}$ By reducing this stress, while energy supply remained unchanged, one might anticipate improved maternal nutritional status, transmitting health benefits to the next generation. But the outcome was different: a pioneering evolutionary analysis by Gibson and Mace concluded that the 'energy saved' by the installation of village water taps enhanced maternal fertility, which was associated with worsening childhood malnutrition. They suggested that the outcome might have been better if the intervention had included a family planning component.

This example highlights the potential benefits of an evolutionary perspective in public health. Human physiology and behaviour have been selected to transmit genes to future generations. Health is sometimes compromised in favour of immediate survival or reproduction, and particularly so under conditions of deprivation and environmental 
harshness. Public health has benefitted substantially from incorporating a life-course perspective, capable of integrating the impacts of physical, biological and societal stresses or stimuli at different life stages. ${ }^{3-6}$ Evolutionary approaches could extend these benefits, providing new insight into the health-consequences of efforts to change behaviour patterns or the environment.

In 1973, Dobzhansky observed that 'nothing in biology makes sense except in the light of evolution' ${ }^{7}$ Through the $20^{\text {th }}$ century, evolutionary approaches permeated most areas of biological enquiry, and are increasingly employed by policy makers in agriculture and fisheries management. ${ }^{8,9}$ Surprisingly, however, an evolutionary perspective on medicine only emerged recently.

A key benefit is the availability of solid overarching theory. Most natural sciences have a strong theoretical basis, e.g. quantum theory in physics, molecular theory in chemistry. ${ }^{10}$ Evolution is also a 'basic science', ${ }^{11}$ and it is no exaggeration to suggest that its application in medicine could revolutionize the discipline. In the $19^{\text {th }}$ century, for example, pre-Darwinian biology was mainly descriptive. Variability was well documented, but poorly understood.

Medicine remains largely pre-evolutionary - excelling in description and mechanistic explanations, but only beginning to explain variability in disease vulnerability across individuals and populations. Evolutionary theory generates testable hypotheses regarding how organisms should respond to environmental stimuli, and these hypotheses are widely supported in diverse species including humans. ${ }^{12-14}$ 
To date, evolutionary medicine has primarily aimed to go beyond understanding how people get sick by considering why bodies are vulnerable to disease. This helps understand why people present at clinics, but may not help prevent illness from developing. Building on earlier work, ${ }^{15}$ we argue that evolutionary approaches could benefit outcomes most directly in the arena of public health.

In particular, they may help understand the health-impact of ecological change, whether this relates to non-human or societal factors. Traditionally, public health efforts targeted risk factors related to pathogens. To prevent disease transmission hygiene and sanitation were improved, as were nutrition and living conditions to promote resilience. Although pathogens remain a major disease source, the global burden of ill-health is shifting towards noncommunicable diseases (NCDs), where individuals' constitution and behaviour are key to susceptibility and prevention. ${ }^{16}$ Whilst some overt risk factors have been identified (e.g. tobacco, dietary trans-fats, carcinogens), many lie nested within 'normal lifestyles' (e.g. enjoyable behaviours) or the 'normal range' of physiological variability (e.g. patterns of growth and maturation). In turn, our lifestyles are shaped by broader societal phenomena, connecting health with cultural and political factors. ${ }^{17}$ By shedding more light on how physiology and behaviour respond to such compound stresses, evolutionary approaches could improve societal efforts to prevent NCDs, just as they already help reduce the burden of infectious disease. ${ }^{18}$

This article has three objectives. First, we describe two primary components of evolutionary theory - natural selection and population genetics, and life history theory which provides a predictive framework for investigating plasticity. Second, we briefly discuss the physiological 
and behavioural mechanisms that underpin plasticity, to elucidate how our evolved biology responds to environmental change. Third, we show how mathematical models could help predict the effects of interventions prior to their implementation. Three other papers in this series focus in more detail on reproduction, human-microbe interactions and nutrition. ${ }^{19-21}$

\section{Evolution, heritability and genetics}

Darwin's and Wallace's theory of natural selection provided new insight into how ancestral environments shape contemporary biological variability. ${ }^{22}$ The theory proposed that traits varied, that this variability had a heritable component, and that organisms producing more offspring transmitted their traits with greater frequency to subsequent generations. Over time, a lineage acquires the genes and phenotypes of those reproducing most successfully. ${ }^{23}$ Though simply a 'purposeless algorithm', ${ }^{24}$ natural selection shapes traits to enhance genetic fitness. ${ }^{25}$ In Darwin's time, scientific understanding of the mechanisms of heredity was rudimentary. Modern genetics emerged from the rediscovery of Mendel's work in the late $19^{\text {th }}$ century, laying the foundation of the modern evolutionary synthesis. ${ }^{26}$

That genetic variants influence disease risk is now well-established, ${ }^{27}$ prompting interest in gene-based 'personalized medicine'. Concerning treatment, ethnic differences in the frequency of genes that influence drug metabolism have attracted attention. ${ }^{28}$ Looking at pathogens in combination with their human hosts, most clinicians will be familiar with the evolutionary emergence of new infectious diseases, such as HIV, Hantaviruses, SARS and Ebola; ${ }^{29}$ with the possibility that imperfect vaccines can make pathogens more virulent; ${ }^{30}$ 
and with the striking threats posed by the evolution of drug- or antibiotic-resistant strains of some pathogens. ${ }^{31}$

Genetic variability is also relevant to public health, in particular for understanding population variability in physiology. For example, where malaria is prevalent it has selected for protective haemoglobin variations, though these may also generate health penalties such as high prevalences of haemoglobinopathies deriving from autosomal recessive genes in malaria-exposed populations. ${ }^{32}$ Importantly, high-fitness genotypes do not maximize pathogen defence, but rather optimize trade-offs with other biological functions, ${ }^{33}$ as described below. Several evolutionary theories have been proposed for ethnic genetic differences in NCD susceptibility (Supplementary Table 1), though the supporting evidence is variable.

However, $\sim 85 \%$ of human genetic variation occurs within rather than between populations. ${ }^{34}$ Pedigree and twin studies indicate that NCDs cluster within families, ${ }^{35,36}$ and a key aim of the Human Genome Project was to identify individual contributing alleles. ${ }^{37}$ As yet, the additive effect of common alleles, potentially favoured though selection, explains little variance in NCD risk. Instead, rare deleterious alleles that evolved too recently to have been selected out of the gene pool seem better genetic predictors of ill health. ${ }^{38}$ It is often suggested that natural selection has ceased in humans, but a more realistic scenario is that it has accelerated in concert with the population boom of the last 10,000 years, increasing the number of new mutations. ${ }^{39}$ 
Our genes clearly contribute to individual variability in disease susceptibility, while genetic analyses can help identify biological pathways to be targeted by pharmaceutical treatment. ${ }^{40}$ Nevertheless, the significance of genotypes for public health is limited by our inability to target them directly for interventions. Genes do not change within generations, and with few exceptions, such as the use of pre-implantation diagnosis in assisted reproduction to screen out rare deleterious alleles, efforts to influence allele frequencies across generations are ethically unacceptable. ${ }^{41}$ We therefore turn to a second component of biological variability that is highly amenable to intervention: plasticity.

\section{Evolution and plasticity}

Plasticity refers to the range of phenotypes potentially elicited by the environment from a single genotype. Plasticity has several different dimensions including behaviour, physiology and development, and responses that range from the momentary to the trans-generational. The primary evolutionary approach to plasticity is 'life history theory', which aims to predict how developing organisms respond to environments to maximize chances of survival and reproduction..$^{42}$

Life history theory provides a framework for understanding how organisms make physiological and behavioural 'decisions' - though behavioural decisions need have no basis in conscious deliberation. Patterns of growth, maturation, reproduction and metabolism account for substantial variation in the risk of NCDs and diverse cancers, but the very 'normality' of these traits has hindered deeper understanding of how they contribute to the aetiology of ill-health, and how they might be targeted by public health programmes. 
Crucially, their associations with health outcomes may also differ substantially between high-income and low-/middle-income settings. Life history theory can help explain this complexity, and offers a holistic framework that can integrate different components of human health.

\section{Life history theory}

Life history theory was developed to predict the coordinated evolution of the traits contributing directly to fitness: age/size at maturation, number/size of offspring, number of reproductive events per life, and aging and lifespan. It views the evolution of these traits as the product of interactions between (a) 'intrinsic constraints' and 'trade-offs' - features inherited or acquired during development - and (b) 'extrinsic factors' in the environment that affect mortality risk and resource availability. It then considers how extrinsic factors shape the combination of intrinsic traits to maximize fitness. ${ }^{42,43}$

Life history theory models phenotypic evolution in general. Everything in biology has both a mechanistic explanation that answers the question, how does this work?, and an evolutionary explanation that answers the questions, how did this get here and what maintains its state? While these questions can be considered over the long-term, to understand why a species has particular traits, they can also be considered within the lifecourse, to understand why individual organisms respond to environmental factors in particular ways. Plastic responses to environmental stimuli include physiological adaptations implemented by homeostatic feedback loops that can react in seconds or minutes; acclimations (e.g. adjustments to altitude) that can react in days to weeks through changes 
in the set-points of feedback loops; and finally, developmental plasticity, where reactions usually last a lifetime. ${ }^{44,45}$

The medical significance of plasticity is most apparent in the 'developmental origins of adult health and disease'.4,46 Variation in early-life experience has many consequences, for example under-nutrition in utero increases risk of NCDs in late life, ${ }^{4,46}$ delivery by C-section increases risk of asthma and obesity, ${ }^{47,48}$ and receiving more antibiotic treatments before two years increases risk of obesity and allergies. ${ }^{49,50}$

While consistent with genetic theories of evolution, the predictions of life history theory explain much more phenotypic variation, thus justifying its simplifications. Because physicians and public health professionals deal with phenotypes, they can gain substantially from a theory that predicts phenotypic states and how they are expected to change over an individual's life-course. Going beyond 'standard care', understanding of each individual's ongoing life history could guide personalized decisions concerning the prevention, diagnosis, and treatment of disease.

\section{Trade-offs and reaction norms}

Two key concepts in life history theory are trade-offs and reaction norms. A trade-off occurs whenever a change in one trait that increases fitness is connected to a change in another trait that decreases fitness. The major functions involved in trade-offs are maintenance, growth, reproduction, and defence, in each of which energy can be invested (Supplementary online Figure 1). Differential investment between these functions is shaped both by 
resource availability, and by extrinsic mortality risk, of which key components in humans include infectious disease, poverty/deprivation and violence/conflict. In general, organisms with high mortality risk invest in rapid growth and reproduction at the expense of maintenance and defence, in which organisms with low mortality risk invest more. Thus the life histories of species occupying contrasting environments diverge, creating a continuum from small, 'fast-living' short-lived species to large, 'slow-living' long-lived species (Figure 1).

This continuum also characterises individual variation within species, including humans. Natural selection has shaped individuals to respond to cues of extrinsic mortality risk and resource availability with phenotypic change that maximize fitness. Specific responses include variation in age and size at maturity, the interval between births, and investment in offspring. The quality of the external environment therefore shapes the entire schedule of growth, maturation, reproduction and aging. This helps explain the profound variability in 'life tables', describing age-specific mortality rates and life expectancies among human populations, highlighting 'slower' and 'faster' life history trajectories within our species (Supplementary online Table 2).

Each individual represents a 'bundle' of many trade-offs. For example, the trade-off between reproduction and survival (maintenance/defence) shapes the rate of aging and NCD risk. Trade-offs are crucial for physicians and public health planners because they force us to recognize that we cannot change one trait without also changing others, sometimes for the worse. Two trade-offs especially relevant to public health, namely immune function versus growth, and reproduction versus longevity, are summarized in Panel 1. 
The second key concept, the reaction norm, describes the spectrum of phenotypes produced by a single genotype across a range of environmental conditions. Life history theory predicts the evolution of reaction norms themselves, as well as the state of traits expressed in specific environments. This approach clarifies how nature always interacts with nurture during development to produce the state of the observed organism. Examples of human reaction norms include age and size at maturity ${ }^{51}$ and variation in inter-birth interval induced by changes in nutritional status (Panel 2, Figure 2).

Several issues are important when applying life history theory to humans. First, our sociality connects the life histories of multiple individuals. Humans demonstrate 'cooperative breeding', whereby several individuals may contribute to a 'pooled energy budget' for investment in offspring. ${ }^{52}$ Sociality can also expose individuals to stresses, such as social hierarchy and inter-group conflict. ${ }^{18}$ Second, cultural values that influence behaviour may themselves evolve over time, examples including attitudes to wealth, risk, or the costs and benefits of raising children. ${ }^{53,54}$ 'Cultural' goals may be pursued at the expense of genetic fitness. Third, evolved behaviour need not necessarily benefit health or fitness, an example being the use of narcotic substances that trigger 'reward centres' in the brain whilst compromising physiological function. ${ }^{55}$

One might question whether humans in affluent environments still experience trade-offs. Energy can be stored outside the body in material form or social relationships, or in adipose tissue. ${ }^{56}$ Yet although wealthy humans acquire energy to invest in each of growth, health and reproduction, subtle trade-offs are both predicted ${ }^{57}$ and observed, for example between family size and the growth rate of individual children. ${ }^{58}$ Similarly, while obesity might suggest 
a surfeit of calories, it is better considered a state of 'metabolic perturbation', where perturbed insulin dynamics provoke 'cellular starvation'. ${ }^{59}$ Finally, some trade-offs involve conflicts in signalling among immune cells or in gene expression networks, and are mediated not by energy but by information. These exist regardless of nutritional status.

The specific 'decisions' that constitute each individual's life history trajectory are enacted at levels that include physiology and behaviour. Many of the relevant mechanisms are already well understood to shape disease risk. What we emphasize here is that these are the same mechanisms that permit adaptation through plasticity to ecological stresses. Both hormonal and behavioural plasticity represent mechanisms of 'risk management' that are inherently sensitive to physical and societal stimuli. ${ }^{56}$

\section{Life history plasticity and hormones}

Hormones allow organisms to respond to both endogenous and exogenous environmental factors by modifying cell functions variably across tissues and organs. ${ }^{60}$ Hormones are now recognized to generate multiple physiological effects, a scenario known as pleiotropy. ${ }^{60}$

For example, insulin plays a key role in allocating energy across competing physiological functions. Conventionally, clinicians think of insulin as responsible for regulating blood glucose, and variability in its production or activity is central to the constellation of diseases

grouped as diabetes. ${ }^{61}$ However, its total metabolic profile is far more complex, and it affects diverse functions in tissue-specific ways. ${ }^{62}$ Via receptors in the brain, insulin modulates the regulation of peripheral metabolism, including appetite, reproductive 
function, thermoregulation and adiposity. ${ }^{62,63}$ Within the brain, insulin also regulates cognitive functions such as learning and memory. ${ }^{64}$ While muscle insulin resistance increases risk of diabetes, it also allows the diversion of fuel to other tissues. ${ }^{62}$

Leptin, secreted by adipose tissue, signals the magnitude of energy stores to the brain but also has broader functions, contributing to the regulation of reproduction, cognitive function and immune function. ${ }^{65,66}$ For example, leptin influences the functions of T-cells, monocytes, macrophages and natural killer cells, as well as the release and expression of cytokines and other inflammatory markers, while these molecules likewise contribute to the regulation of energy balance. ${ }^{66}$ While early linear growth benefits long-term health and human capital, ${ }^{67}$ the association between low leptin and mortality in malnourished children indicates the short-term survival value of body fat. ${ }^{68}$

Another influential hormone is cortisol, produced by the adrenal glands in response to diverse types of stress including illness, trauma, fear, pain and psychosocial stress. It too impacts diverse metabolic activities, for example suppressing immune function while increasing blood pressure and blood glucose. ${ }^{69}$

In each case, therefore, these hormones implement the allocation of energy between life history functions. Whilst such plasticity may be adaptive, especially in the context of reproduction (see paper 3 ) it may also impose metabolic costs, accelerating the rate of aging (see Paper 4). Furthermore, human societies generate stresses for which their biology is unprepared or 'mismatched', ${ }^{70}$ such as pollutants, processed foods and sedentary environments. 
Many trade-offs pertain to individual organisms. However, mammalian reproduction inherently brings the life history strategies of two generations together, through placental nutrition and lactation. This interaction may be characterized as a 'tug-of-war' over maternal metabolic resources, ${ }^{71}$ for the energy allocation 'decisions' optimal for maternal fitness may not be those that maximize offspring fitness. In such 'parent-offspring conflict' (Supplementary online Panel 1), ${ }^{72}$ the hormones reviewed above now function as signals between individuals, and each party can not only 'read' signals of the other, but also potentially manipulate them through their own hormonal secretions. ${ }^{73}$ The consequences of this 'tug-of-war' are expressed in several outcomes relevant to public health, including the prevalence of low birth weight, the incidence of colic, the duration of breast-feeding, and the management of infant sleep. ${ }^{73-75}$ The tug-of-war can itself be targeted by interventions, for example a randomized trial showed that promoting relaxation in breast-feeding mothers was associated with faster growth in their offspring. ${ }^{76}$

\section{Life history theory and behaviour}

Conventionally, public health models of behaviour emphasize 'purpose' and 'individual autonomy', in other words 'how a person thinks rather than ... what he or she does' ${ }^{77}$ hence campaigns often target conscious deliberation. In contrast, life history theory makes predictions about behaviour itself, and makes no assumptions about whether decisions are made consciously or unconsciously. In other species, this question does not arise. Some conscious thought may simply provide post-hoc rationalization - more consequence of behaviour than cause. 
Particularly in high-income low-fertility populations, contemporary behaviour is not maximizing fitness. This is partly because of cultural preferences (for wealth, social status, health, hedonic pleasure) that evolve independently of genes, and partly because of 'adaptive lag' whereby environments change more rapidly than human biology. ${ }^{78}$ But we can still use evolutionary principles to understand associations between behaviour and health outcomes.

In long-lived species such as humans, which produce offspring at regular intervals, the value of investing in somatic maintenance and future reproduction is expected to vary with ecological conditions. Higher extrinsic mortality risk favours diverting energy from maintenance to earlier reproductive effort. Why stint on reproduction if one is likely to die soon? Conversely, lower mortality risk favours higher investment in somatic maintenance, which may benefit future reproduction as well as longevity. Variation in mortality risk can therefore help explain both within- and between-population variation in behaviours relevant to public health, including reproductive 'decisions' and engagement in 'risky' behaviours (Supplementary online Panel 2).

For example, reproductive timing varies in association with environmental harshness. In high-income countries, lower socio-economic position (SEP) correlates with earlier reproduction, and poorer health status may be an important explanatory variable. In England (2009-2011), living in areas with the highest deprivation (measured in deciles) was associated with 7 and 9 years shorter life expectancy for women and men respectively, compared with those in the least-deprived areas. Equivalent differences in healthy life 
expectancy were twice as large. ${ }^{79}$ Early reproduction in low SEP women may therefore reflect both their lower expectancy of healthy life and the absence of benefits of waiting, for they typically have fewer opportunities to capitalize on educational and career opportunities. A link between deprivation and early age at first birth also holds across populations. ${ }^{80}$ Greater energy investment in reproduction indicates lower investment in homeostasis (Panel 1), and may contribute to elevated NCD risk in low SEP populations. ${ }^{18}$

In behavioural terms, lack of investment in self-preservation may be mediated by 'time preferences', where short-term gains are favoured over long-term rewards. ${ }^{81}$ For example, individuals unable to assume a long, healthy life lies ahead are expected to 'discount the future' and prioritize immediate rewards, whether through conscious or subconscious mechanisms (Supplementary online Panel 2). Individuals more oriented to the present report more risk-prone attitudes than those oriented to the future. ${ }^{82}$ However, the trade-off between longevity and reproduction can also be exploited for health benefit by interventions designed to appeal to personal 'attractiveness', as demonstrated for diet and cancer risk. ${ }^{83,84}$

Extrinsic mortality risk therefore predicts many 'unhealthy behaviours' (smoking, drug consumption, poor diet, risky sexual behaviours) as well as lower commitment to healthy behaviours such as physical activity. Such unhealthy behaviours are consistently linked with lower SEP in high income countries, ${ }^{85}$ and this relationship appears to be mediated through higher perceptions of extrinsic mortality risk experienced by lower SEP individuals. ${ }^{86}$ Note that though such behaviours contribute to socio-economic health inequalities, they are not sufficient to entirely explain observed differences in life expectancy by SEP, indicating that 
structural and economic constraints are also important. Public health campaigns targeting such unhealthy behaviours might therefore have greater success if supported by efforts to reduce deprivation and increase access to health-care. Currently, however, medical treatment in some countries may be withheld from those who smoke or are obese.

\section{Mathematical modelling}

A strength of life history theory is that it can be expressed in terms of equations, enabling mathematical modelling. This may allow potential benefits and costs of interventions to be considered before their implementation in vivo. While models inevitably have limitations related to the assumptions involved, they may flag up in advance issues meriting more attention. While applicable to many contexts - e.g. predicting reaction norms or examining host-pathogen dynamics - they are particularly valuable for understanding parent-offspring dynamics, where life histories interact.

To illustrate this we briefly consider the challenge of reducing child malnutrition, a major global health problem. ${ }^{87}$ Logic suggests a simple solution: increased energy supply. Since low birth weight contributes to subsequent malnutrition, logic also suggests that interventions should target pregnant mothers. Protein-energy supplementation programmes have thus been provided for pregnant mothers in several countries. ${ }^{88}$

As the Ethiopian example demonstrated, however, mothers face a trade-off between investing in current versus potential future offspring. ${ }^{89}$ Additional energy might either support growth and survival of the former, or accelerate production of the latter. Assuming 
that maternal metabolism has been selected to optimize lifetime reproductive fitness, models can predict the optimal energy allocation (Panel 3, Figure 3). Even simple models can clarify the issues and suggest qualitative predictions.

Our model suggests that maternal supplementation can lead both to improved offspring survival and to a shorter period of dependency, increasing maternal fecundity. The balance between these effects, however, differs markedly according to the duration of the intervention. A short-term boost in resources promotes offspring growth and survival, whereas a long-term improvement primarily benefits maternal fecundity with little benefit for the size of individual offspring.

The underlying reason is that mothers must balance the benefit of prolonged care for the current offspring against the risk that she will die and lose the opportunity to produce additional children. A short-term energy windfall increases the benefit of extending care for the current offspring, while leaving the mother's long-term prospects unchanged. By contrast, a long-term improvement in resources increases the chances of future reproductive success, devaluing investment in the current offspring. Once again, this highlights how reproductive fitness may take priority over the health of individuals.

\section{The emerging field of evolutionary public health}

Life history theory improves understanding of human variability in disease susceptibility, and of how the organized efforts of societies to change behaviour or environments may impact health outcomes. Both physiology and behaviour respond to ecological stimuli through the 
medium of trade-offs and reaction norms that favour survival and reproduction over health. Both physiology and behaviour have been selected to 'discount the future' in high-risk environments. One key insight is that we should not expect a given intervention to produce identical consequences in populations that contrast in resource availability and extrinsic mortality risk.

This helps understand why poverty and deprivation have such powerful impacts on health and lifespan, and should themselves be a key target for interventions. Experience in early life may impact the entire trajectory of maturation and aging, generating trade-offs between reproduction and homeostasis. ${ }^{18}$ Consequently, programmes targeting individual behaviour might have greater health benefits if linked with broader efforts to combat poverty, deprivation and extrinsic mortality risks. Another key insight is that every individual phenotype reflects an accumulated history of trade-offs. This information could potentially improve the personalization of disease prevention, diagnosis and management.

Evolutionary approaches are likely to be particularly valuable for addressing the challenges of global health, where populations are differentially exposed to multiple metabolic costs deriving from high fertility, diverse infectious diseases, and rapid changes in nutrition and lifestyle. Given such heterogeneity, mathematical modelling could be used to explore the likely costs and benefits of local interventions in silico, before their implementation in vivo. More broadly, evolutionary approaches offer a unique predictive framework with which to understand the basis of human disease and improve the efficacy of public health interventions. 


\section{Contributors}

All authors wrote sections of this report, provided feedback on drafts, and approved the final version.

\section{Funding}

Preparation of this manuscript was facilitated by support of the Arizona State University Centre for Evolution and Medicine provided for an authors meeting. The model of growth was produced through a grant to RAJ and JCW from the Leverhulme Trust. 


\section{Key messages}

\section{What we know}

- Evolutionary theory is likely to improve the efficacy and integration of public health interventions, given its utility in other areas of public policy

- Evolutionary life history theory is integrative, and can inform both physiological and behavioural components of public health interventions

- Based on optimization principles, life history theory allows potential interventions to be modelled using mathematical techniques, identifying likely consequences before implementation in vivo

\section{What we need to know}

- How do predictions from life history theory change when populations occupy affluent and benign environments, and many individuals choose not to produce offspring?

- How should we balance benefits versus costs that appear in different parts of the life-course, such as when interventions promoting early health affect long-term health adversely?

- How can we integrate the insights generated by applying life history theory to plasticity into 'personalized medicine'?

- How can we use life history theory to improve public health campaigns promoting 'behaviour change'? 
Panel 1. Life history theory predicts trade-offs relevant to public health

- Immune function is metabolically costly, ${ }^{90}$ and in children each degree temperature rise from fever increases metabolic rate by $11.3 \%,{ }^{91}$ hence the costs of fighting infections impair child growth. ${ }^{92}$

- This can account for epidemiological associations linking secular declines in infant mortality rate (a proxy for the energy costs of immune function in the survivors) with secular increases in adult height and longevity. ${ }^{93}$ Developmental exposure to infectious diseases shapes the entire life history strategy, and may propagate effects to subsequent generations. ${ }^{94}$

- Another key trade-off is between reproduction and longevity, with several studies showing that parental survivorship declined in proportion to the number of children produced, more strongly in mothers than fathers. ${ }^{95}$ However, the magnitude of this effect varies by living standards, and reproduction may protect against some cancers (see Paper 2). ${ }^{96}$

- Such trade-offs also apply across generations: across 27 sub-Saharan African countries, the odds of child survival fell in relation to the number of offspring produced by the mother. ${ }^{97}$

- Public health programmes targeting infant infections or adult reproduction are thus expected to shape long-term health outcomes and disease susceptibility through influencing these trade-offs. For example, nutritional interventions to resolve stunting may be ineffective unless also reducing the burden of infections and parasites. $^{98}$ 
Panel 2. Reaction norms and the trade-offs that shape them

- Maternal age and size at first birth vary with conditions encountered during growth and development. This plasticity maximizes the potential for reproductive success across the range of environments frequently encountered. Life history theory predicts optimal reaction norms consistent with shifts caused by recent changes in nutrition and mortality risk.

- Figure 2a distinguishes between the plastic developmental reaction to environmental change and the genetic evolution of that reaction (i.e. between nurture and nature).

- The upper curve shows the optimal response to environmental improvement: the reproduction event slides up the reaction norm to the left, occurring earlier. While this represents a developmental response, the shape and position of the reaction norm itself have evolved and are genetically determined.

- The lower curve shows the evolution of that reaction norm. Through demographic and epidemiological transition, infant mortality rates fell as public health and medical efforts decreased the impact of infectious disease. ${ }^{99}$ This drove the entire reaction norm down and to the left, resulting in a further decrease in age at first birth and a modest decrease in maternal size.

- Why does the reaction norm change in this way? One major trade-off affecting human maturation relates infant mortality to maternal age (Figure $\mathbf{2 b}$ ). As infant mortality declines, mothers are selected to have their first baby earlier.

- Here, cultural evolution (improved health care) is interacting with biological evolution. It is important to understand that efforts of physicians and public health workers in the interest of promoting health may also shape human evolution itself. 
Panel 3. An optimization model of maternal nutritional supplementation programmes

- To assess the consequences of supplementing mothers to improve the growth of their offspring, we consider a mother producing single offspring sequentially.

- The mother accrues resources (energy) to invest in offspring growth at a rate $r$ per unit time. She is also exposed to a mortality risk $m$ per unit time. The decision she faces is how long to support each offspring before producing the next. Longer support means more resources for the offspring, but greater risk the mother will die before the offspring reaches independence.

- We assume that maternal death prior to independence leads to offspring death, whereas there is no risk of offspring death while the mother survives to care for it.

- Following independence, the offspring survival depends upon the resources it received. We assume that some minimum level of resources is required for viability; beyond this, survival prospects increase with resources, but at an ever-diminishing rate (see supplementary online material).

- Given these assumptions, we can determine the optimal duration of support that maximizes the mother's expected lifetime fitness, and the resulting size and viability of her offspring (Figure 3). We can also ask how these outcomes change if we alter the level of resources available, either during the period of dependency of the current offspring, or throughout the remainder of the mother's life.

- The results show that whereas a short-term increase in maternal resources improves offspring growth and survival, a long-term increase in resource availability serves chiefly to promote greater fecundity in the mother, with little benefit for the size of individual offspring. 


\section{References}

1. Winslow C. The untilled field of public health. Mod Med 1920; 2: 183-91.

2. Gibson MA, Mace R. An energy-saving development initiative increases birth rate and childhood malnutrition in rural Ethiopia. PLoS Med 2006; 3(4): e87.

3. Kuh D, Ben-Shlomo Y. A life course approach to chronic disease epidemiology, 2nd edition. Oxford: Oxford University Press; 2004.

4. Barker DJ. Fetal and infant origins of adult disease. London: British Medical Journal; 1992.

5. Krieger N, Davey Smith G. "Bodies count," and body counts: social epidemiology and embodying inequality. Epidemiologic reviews 2004; 26: 92-103.

6. Marmot M. Social determinants of health inequalities. Lancet 2005; 365(9464): 1099104.

7. Dobzhansky T. Nothing in biology makes sense except in the light of evolution. Am Biol Teacher 1973; 35(3): 125-9.

8. Jørgensen C, Enberg K, Dunlop ES, et al. Ecology: managing evolving fish stocks. Science 2007; 318(5854): 1247-8.

9. Wade N. Green revolution (I): a just technology, often unjust in use. Science 1974; 186(4169): 1093-6.

10. Wilson EO. Consilience: the unity of knowledge. London: Little, Brown and Co.; 1998.

11. Nesse RM, Bergstrom CT, Ellison PT, et al. Evolution in health and medicine Sackler colloquium: Making evolutionary biology a basic science for medicine. Proc Natl Acad Sci U S A 2010; 107 Suppl 1: 1800-7.

12. Stearns SC, Ackermann M, Doebeli M, Kaiser M. Experimental evolution of aging, growth, and reproduction in fruitflies. Proc Natl Acad Sci U S A 2000; 97(7): 3309-13.

13. Winterhalder B, Smith EA. Analyzing adaptive strategies: Human behavioral ecology at twenty-five. EvolAnthropol 2000; 9: 51-72.

14. Nettle D, Gibson MA, Lawson DW, Sear R. Human behavioral ecology: current research and future prospects. Behav Ecol 2013; 24(5): 1031-40.

15. Omenn GS. Evolution in health and medicine Sackler colloquium: Evolution and public health. Proc Natl Acad Sci U S A 2010; 107 Suppl 1: 1702-9.

16. World Health Organization. Global status report on noncommunicable diseases 2010. Geneva: World Health Organization; 2011.

17. Doyal L, Pennell I. The political economy of health. London: Pluto Press; 1979.

18. Wells JC. The metabolic ghetto: an evolutionary perspective on nutrition, power relations and chronic disease. Cambridge: Cambridge University Press; 2016.

19. Rook G, Backhed F, Levin B, McFall-Ngai M, McLean A. Evolution, human-microbe interactions and life history plasticity. Lancet 2016; in series.

20. Jasienska G, Bribiescas RG, Furberg A-S, Helle S, Nunez-de la Mora A. Human reproduction and health: an evolutionary perspective. Lancet 2016; in series.

21. Wells JC, Watve M, Leonard WR, Victora CG, Kirkwood TB. An evolutionary perspective on nutrition, ageing and non-communicable diseases. Lancet 2016; series paper. 22. Darwin C. On the origins of species by means of natural selection. London: Murray; 1859.

23. Dobzhansky T, Boesinger E. Human culture: a moment in evolution. New York: Columbia University Press; 1983.

24. Dennett DC. Darwin's dangerous idea. London: Penguin; 1995. 
25. Hamilton WD. The genetical evolution of social behaviour. I. J Theor Biol 1964; 7(1): $1-16$.

26. Jablonka E, Lamb MJ. Evolution in four dimensions: genetic, epigenetic, behavioral, and symbolic variation in the history of life. Cambridge, MA: MIT Press; 2005.

27. Frazer KA, Murray SS, Schork NJ, Topol EJ. Human genetic variation and its contribution to complex traits. Nat Rev Genet 2009; 10(4): 241-51.

28. Ono C, Kikkawa $H$, Suzuki A, et al. Clinical impact of genetic variants of drug transporters in different ethnic groups within and across regions. Pharmacogenomics 2013; 14(14): 1745-64.

29. Jones KE, Patel NG, Levy MA, et al. Global trends in emerging infectious diseases. Nature 2008; 451(7181): 990-3.

30. Gandon S, Mackinnon MJ, Nee S, Read AF. Imperfect vaccines and the evolution of pathogen virulence. Nature 2001; 414(6865): 751-6.

31. Arias CA, Murray BE. Antibiotic-resistant bugs in the 21st century--a clinical superchallenge. N Engl J Med 2009; 360(5): 439-43.

32. Weatherall DJ. The inherited diseases of hemoglobin are an emerging global health burden. Blood 2010; 115(22): 4331-6.

33. Ardia DR, Parmentier HK, Vogel LA. The role of constraints and limitation in driving individual variation in immune response. Funct Ecol 2011; 25: 61-73.

34. Barbujani G, Magagni A, Minch E, Cavalli-Sforza LL. An apportionment of human DNA diversity. Proc Natl Acad Sci U S A 1997; 94(9): 4516-9.

35. Goldgar DE, Easton DF, Cannon-Albright LA, Skolnick MH. Systematic populationbased assessment of cancer risk in first-degree relatives of cancer probands. J Natl Cancer Inst 1994; 86(21): 1600-8.

36. Seaquist ER, Goetz FC, Rich S, Barbosa J. Familial clustering of diabetic kidney disease. Evidence for genetic susceptibility to diabetic nephropathy. N Engl J Med 1989; 320(18): 1161-5.

37. Collins FS, Mansoura MK. The Human Genome Project. Revealing the shared inheritance of all humankind. Cancer 2001; 91(1 Suppl): 221-5.

38. Cohen JC, Kiss RS, Pertsemlidis A, Marcel YL, McPherson R, Hobbs HH. Multiple rare alleles contribute to low plasma levels of HDL cholesterol. Science 2004; 305(5685): 869-72.

39. Cochran G, Harpending H. The 10,000 year explosion: how civilization accelerated human evolution. New York: Basic Books; 2009.

40. Hirschhorn JN. Genomewide association studies--illuminating biologic pathways. $N$ Engl J Med 2009; 360(17): 1699-701.

41. Sermon K, Van Steirteghem A, Liebaers I. Preimplantation genetic diagnosis. Lancet 2004; 363(9421): 1633-41.

42. Stearns SC. The evolution of life histories. Oxford: Oxford University Press; 1992.

43. Roff DA. Evolution of life histories: theory and analysis. London: Chapman and Hall; 1992.

44. Lasker GW. Human biological adaptability. The ecological approach in physical anthropology. Science 1969; 166(3912): 1480-6.

45. Wells JC, Stock JT. The biology of the colonizing ape. Am J PhysAnthropol 2007; Suppl 45: 191-222.

46. Gluckman PD, Hanson MA, Cooper C, Thornburg KL. Effect of in utero and early-life conditions on adult health and disease. N Engl J Med 2008; 359(1): 61-73. 
47. Thavagnanam S, Fleming J, Bromley A, Shields MD, Cardwell CR. A meta-analysis of the association between Caesarean section and childhood asthma. Clin Exp Allergy 2008; 38(4): 629-33.

48. Darmasseelane K, Hyde MJ, Santhakumaran S, Gale C, Modi N. Mode of delivery and offspring body mass index, overweight and obesity in adult life: a systematic review and meta-analysis. PLoS One 2014; 9(2): e87896.

49. Trasande L, Blustein J, Liu M, Corwin E, Cox LM, Blaser MJ. Infant antibiotic exposures and early-life body mass. Int J Obes (Lond) 2013; 37(1): 16-23.

50. Murk W, Risnes KR, Bracken MB. Prenatal or early-life exposure to antibiotics and risk of childhood asthma: a systematic review. Pediatrics 2011; 127(6): 1125-38.

51. Stearns SC, Medzhitov R. Evolutionary medicine. Sunderland: Sinauer Associates; 2015.

52. Hrdy SB. Mothers and others: the evolutionary origins of mutual understanding. Cambridge, MA: Belknap Press; 2009.

53. Alvergne A, Lummaa V. Ecological variation in wealth-fertility relationships in Mongolia: the 'central theoretical problem of sociobiology' not a problem after all? Proc Biol Sci 2014; 281(1796): 20141733.

54. Colleran H, Jasienska G, Nenko I, Galbarczyk A, Mace R. Community-level education accelerates the cultural evolution of fertility decline. Proc Biol Sci 2014; 281(1779): 20132732. 55. Wise RA. Addictive drugs and brain stimulation reward. Annual review of neuroscience 1996; 19: 319-40.

56. Wells JC. The capital economy in hominin evolution: how adipose tissue and social relationships confer phenotypic flexibility and resilience in stochastic environments. Curr Anthropol 2012; 53, Suppl. 6: 466-78.

57. Van Noordwijk AJ, de Jong G. Acquisition and allocation of resources: their inlfuence on variation in life history tactics. Am Nat 1986; 128(1): 137-42.

58. Lawson DW, Mace R. Sibling configuration and childhood growth in contemporary British families. IntJEpidemiol 2008; 37(6): 1408-21.

59. Lustig RH. Childhood obesity: behavioral aberration or biochemical drive? Reinterpreting the First Law of Thermodynamics. NatClinPractEndocrinolMetab 2006; 2(8): 447-58.

60. Finch CE, Rose MR. Hormones and the physiological architecture of life history evolution. The Quarterly review of biology 1995; 70(1): 1-52.

61. Gale EA. Is type 2 diabetes a category error? Lancet 2013; 381(9881): 1956-7.

62. Watve M. Doves, diplomats and diabetes: a Darwinian interpretation of type 2 diabetes and related disorders. New York: Springer; 2013.

63. Kleinridders A, Ferris HA, Cai W, Kahn CR. Insulin action in brain regulates systemic metabolism and brain function. Diabetes 2014; 63(7): 2232-43.

64. McGregor G, Malekizadeh Y, Harvey J. Minireview: food for thought: regulation of synaptic function by metabolic hormones. Molecular endocrinology 2015; 29(1): 3-13.

65. Schneider JE. Energy balance and reproduction. Physiol Behav 2004; 81(2): 289-317.

66. Li J, Li F, Zhao A. Inflammation and leptin. Drug Discovery Today: Dis Mech 2006; 3(3): 387-93.

67. Victora CG, Adair L, Fall C, et al. Maternal and child undernutrition: consequences for adult health and human capital. Lancet 2008; 371(9609): 340-57. 
68. Bartz S, Mody A, Hornik C, et al. Severe acute malnutrition in childhood: hormonal and metabolic status at presentation, response to treatment, and predictors of mortality. $J$ Clin Endocrinol Metab 2014; 99(6): 2128-37.

69. Desborough JP. The stress response to trauma and surgery. British journal of anaesthesia 2000; 85(1): 109-17.

70. Godfrey KM, Gluckman PD, Hanson MA. Developmental origins of metabolic disease: life course and intergenerational perspectives. Trends EndocrinolMetab 2010: -.

71. Moore T, Haig D. Genomic imprinting in mammalian development: a parental tug-ofwar. Trends in genetics : TIG 1991; 7(2): 45-9.

72. Trivers RL. Parent-offspring conflict. AmZool 1974; 14: 249-64.

73. Haig D. Genetic conflicts in human pregnancy. QRevBiol 1993; 68(4): 495-532.

74. Wells JC. Parent-offspring conflict theory, signaling of need, and weight gain in early life. QRev Biol 2003; 78(2): 169-202.

75. Haig D. Troubled sleep: Night waking, breastfeeding and parent-offspring conflict. Evolution, medicine, and public health 2014; 2014(1): 32-9.

76. Shukri NH, Wells JC, Mukhtar F, Lee MS, Fewtrell MS. Mother-infant signalling during breast-feeding: a randomised trial investigating the effects of a relaxation intervention in breastfeeding mothers on breast milk production, breast milk cortisol and infant behaviour and growth. Maternal Child Nutrition 2016; 11(S2): 110.

77. Elder JP, Talavera GA, Gorbach PM, Ayala GX. Theories and structures of public health behavior. In: Scutchfield FD, Keck CW, eds. Principles of public health practice, 2 nd edition. New York: Delmar Learning; 2003: 253-72.

78. Laland KN, Brown GR. Niche construction, human behaviour and the adaptive-lag hypothesis. Evol Anthropol 2006; 15(3): 95-104.

79. Office for National Statistics. Inequality in healthy life expectancy at birth by national deciles of area deprivation: England, 2009-11: Ofice for National Statistics; 2014.

80. Caudell MA, Quinlan RJ. Resource availability, mortality, and fertility: a path analytic approach to global life-history variation. Hum Biol 2012; 84(2): 101-25.

81. Chisholm D. Death, hope and sex. Cambridge: Cambridge University Press; 1999.

82. Schecter DE, Francis CM. A life history approach to understanding youth time preference: mechanisms of environmental risk and uncertainty and atttitudes towrads risk behaviour and education. Hum Nat 2010; 21: 140-64.

83. Hillhouse JJ, Turrisi R. Examination of the efficacy of an appearance-focused intervention to reduce UV exposure. Journal of behavioral medicine 2002; 25(4): 395-409.

84. Whitehead RD, Ozakinci G, Perrett DI. A randomized controlled trial of an appearance-based dietary intervention. Health psychology : official journal of the Division of Health Psychology, American Psychological Association 2014; 33(1): 99-102.

85. Pampel FC, Krueger PM, Denney JT. Socioeconomic disparities in health behaviors. Ann Rev Sociol 2010; 36: 349-70.

86. Pepper GV, Nettle D. Perceived extrinsic mortality risk and reported effort in looking after health: testing a behavioural ecological prediction. Human Nature 2014; 25: 378-92.

87. Black RE, Allen LH, Bhutta ZA, et al. Maternal and child undernutrition: global and regional exposures and health consequences. Lancet 2008; 371(9608): 243-60.

88. Ceesay SM, Prentice AM, Cole TJ, et al. Effects on birth weight and perinatal mortality of maternal dietary supplements in rural Gambia: 5 year randomised controlled trial. BMJ 1997; 315(7111): 786-90. 
89. Smith CC, Fretwell SD. The optimal balance between size and number of offspring. Am Nat 1974; 108: 499-506.

90. Lochmiller RL, Deerenberg C. Trade-offs in evolutionary immunology: just what is the cost of immunity? Oikos 2000; 88: 87-98.

91. Benhariz M, Goulet O, Salas J, Colomb V, Ricour C. Energy cost of fever in children on total parenteral nutrition. ClinNutr 1997; 16(5): 251-5.

92. Lee $\mathrm{G}$, Yori $\mathrm{P}$, Olortegui MP, et al. Comparative effects of vivax malaria, fever and diarrhoea on child growth. Int J Epidemiol 2012; 41(2): 531-9.

93. Crimmins EM, Finch CE. Infection, inflammation, height, and longevity. Proc Natl Acad Sci U S A 2006; 103(2): 498-503.

94. Guyatt HL, Snow RW. Impact of malaria during pregnancy on low birth weight in subSaharan Africa. Clinical microbiology reviews 2004; 17(4): 760-9, table of contents.

95. Penn DJ, Smith KR. Differential fitness costs of reproduction between the sexes. Proc Natl Acad Sci U S A 2007; 104(2): 553-8.

96. Le Bourg E. Does reproduction decrease longevity in human beings? Ageing research reviews 2007; 6(2): 141-9.

97. Lawson DW, Alvergne A, Gibson MA. The life-history trade-off between fertility and child survival. Proc Biol Sci 2012.

98. Panter-Brick C, Lunn PG, Baker R, Todd A. Elevated acute-phase protein in stunted Nepali children reporting low morbidity: different rural and urban profiles. The British journal of nutrition 2001; 85(1): 125-31.

99. Stearns SC, Byars SG, Govindaraju DR, Ewbank D. Measuring selection in contemporary human populations. Nat Rev Genet 2010; 11(9): 611-22.

100. Stearns SC, Koella JC. The evolution of phenotypic plasticity in life-history traits: predictions of reaction norms for age and size at maturity. Evol 1986; 40(5): 893-913. 
Figure 1. Schematic diagram illustrating life history trajectories across a fast-slow continuum. Fast life histories are favoured in environments with high mortality risk, while slow life histories can evolve when mortality risk reduces. These strategies may evolve under natural selection, but physiology can also respond to cues during the life-course through plasticity. The size of the circles is proportional to adult body size, and filled circles indicate individuals that survive to reproduce.

Figure 2. (a) The reaction norm for the age at maturity in relation to body weight in adult females. In any population, the reaction norm allows variability in the response, but the norm itself can also evolve genetically over generations. Reproduced with permission from Stearns and Koella $1986 .{ }^{100}$ (b) The key trade-off that shapes the evolution of this reaction norm: the relationship between maternal age and infant mortality. Reproduced with permission from Stearns $1992 .{ }^{42}$ (See Panel 1).

Figure 3. Results of a model predicting how maternal and offspring traits vary in accordance with ecological conditions, if the goal is to maximize maternal reproductive fitness. A shortterm intervention increasing energy availability increases maternal investment in the current offspring, leading to larger offspring size, and higher chance of offspring survival but has negligible effect on maternal fecundity. In contrast, the benefits of a long-term intervention are primarily captured by the mother through increased fecundity, whereas there is little effect on offspring investment, growth or chance of survival. 
Figure 1

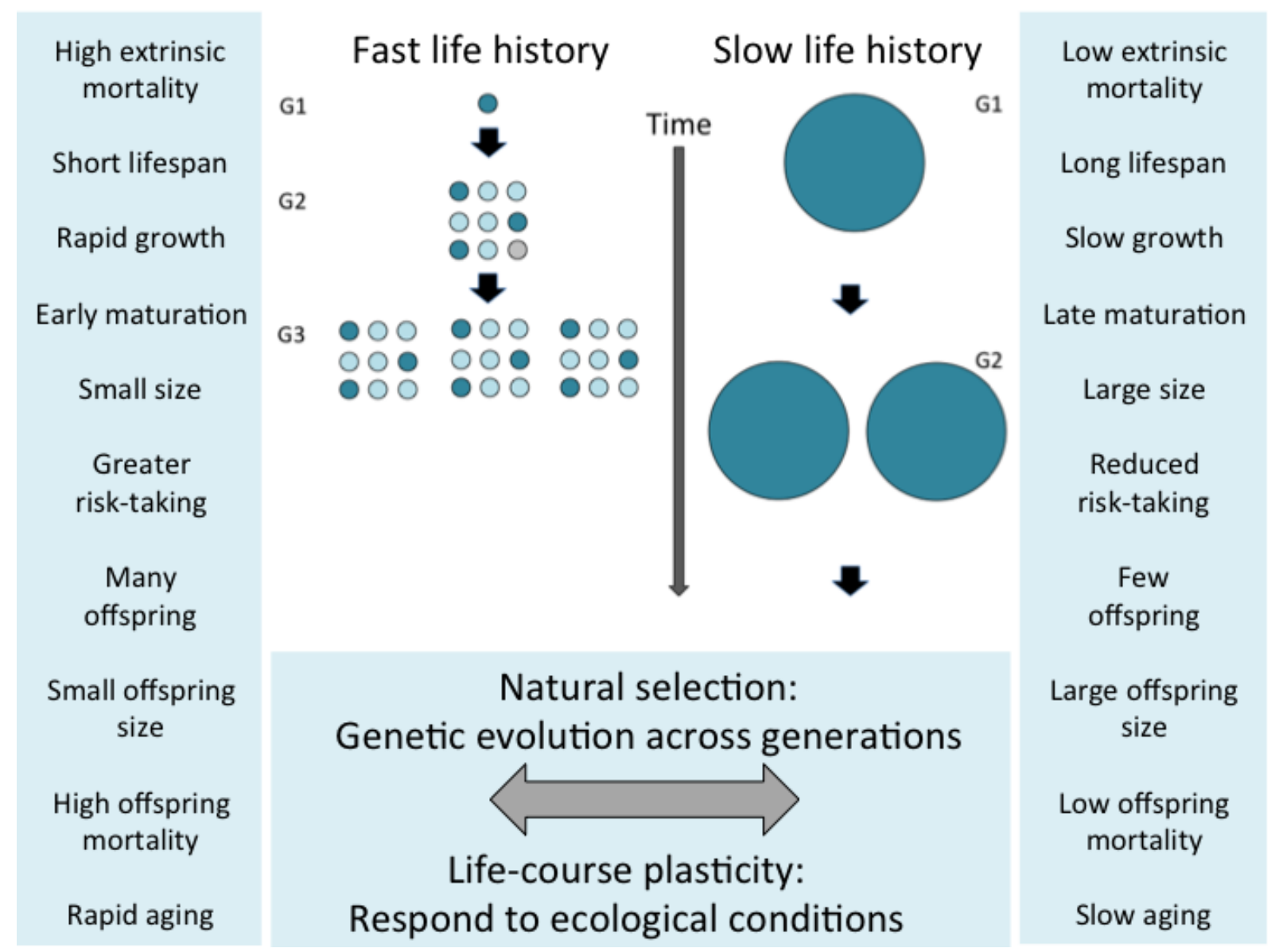


Figure 2
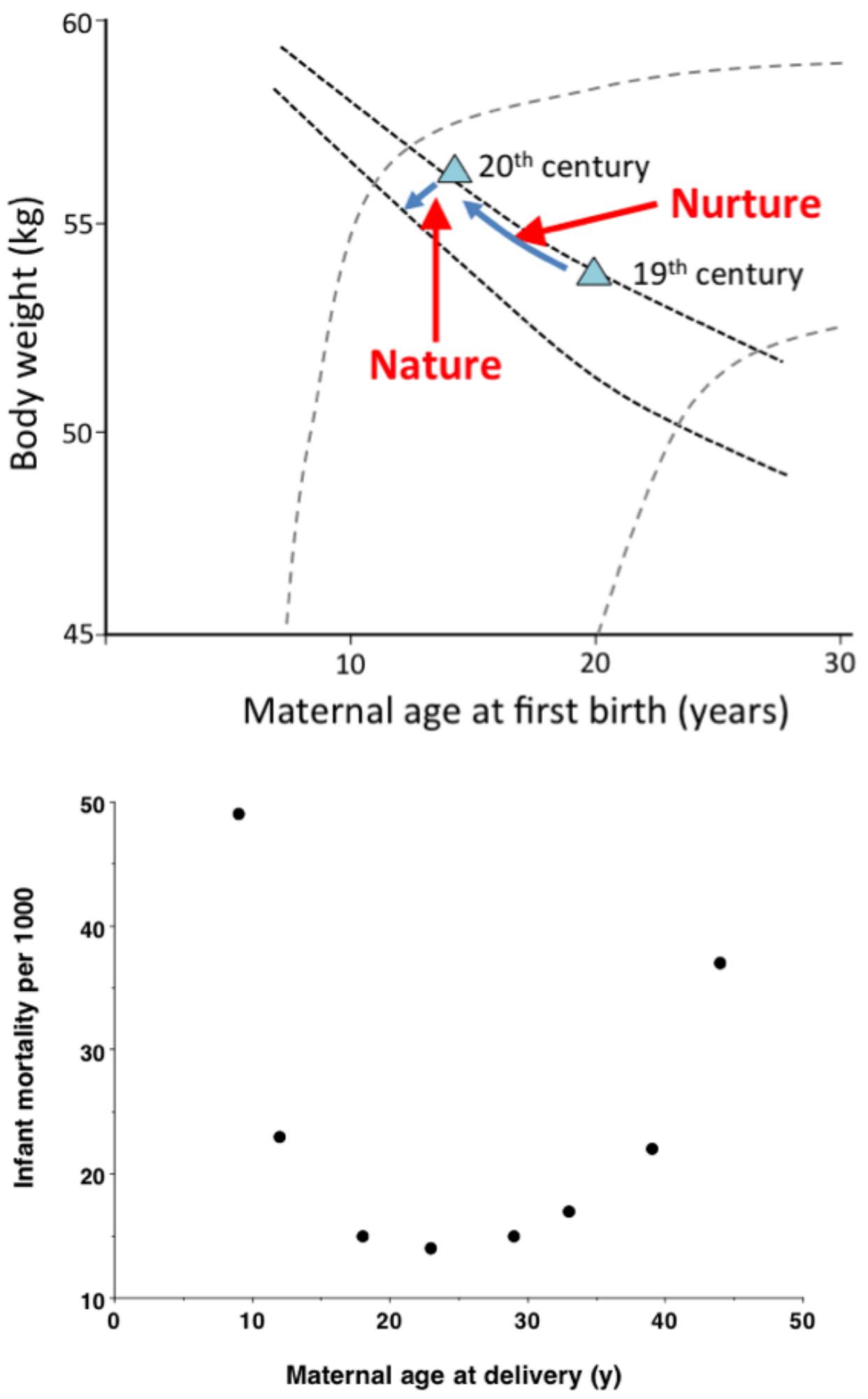
Figure 3
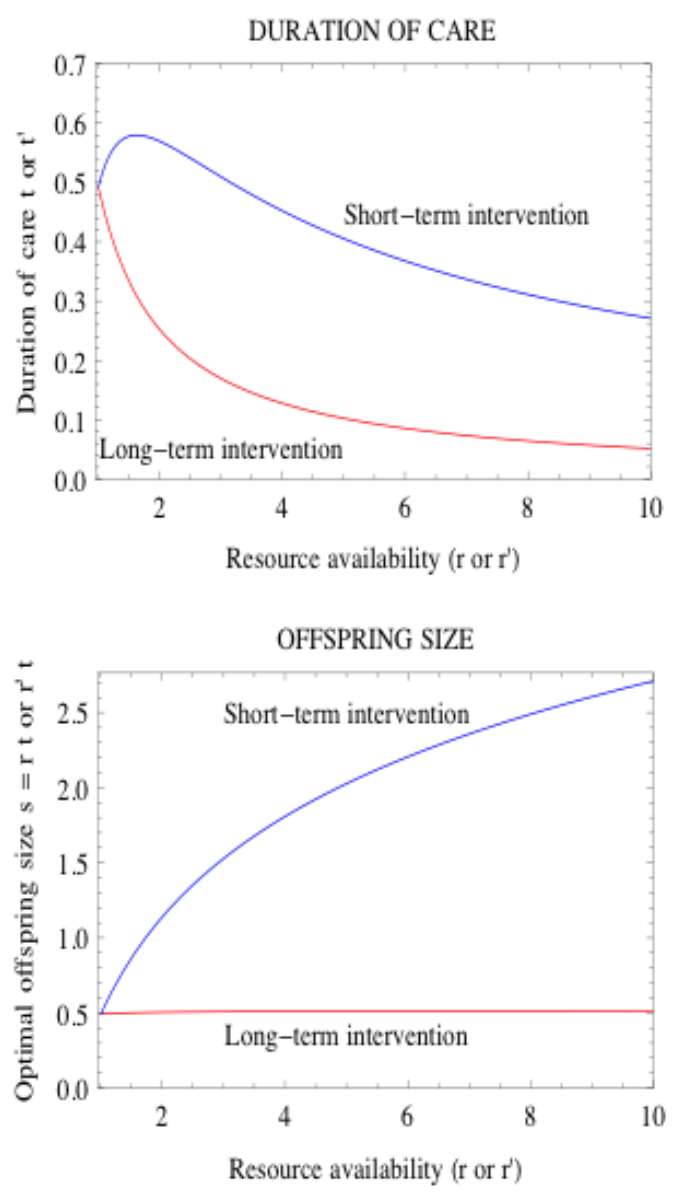
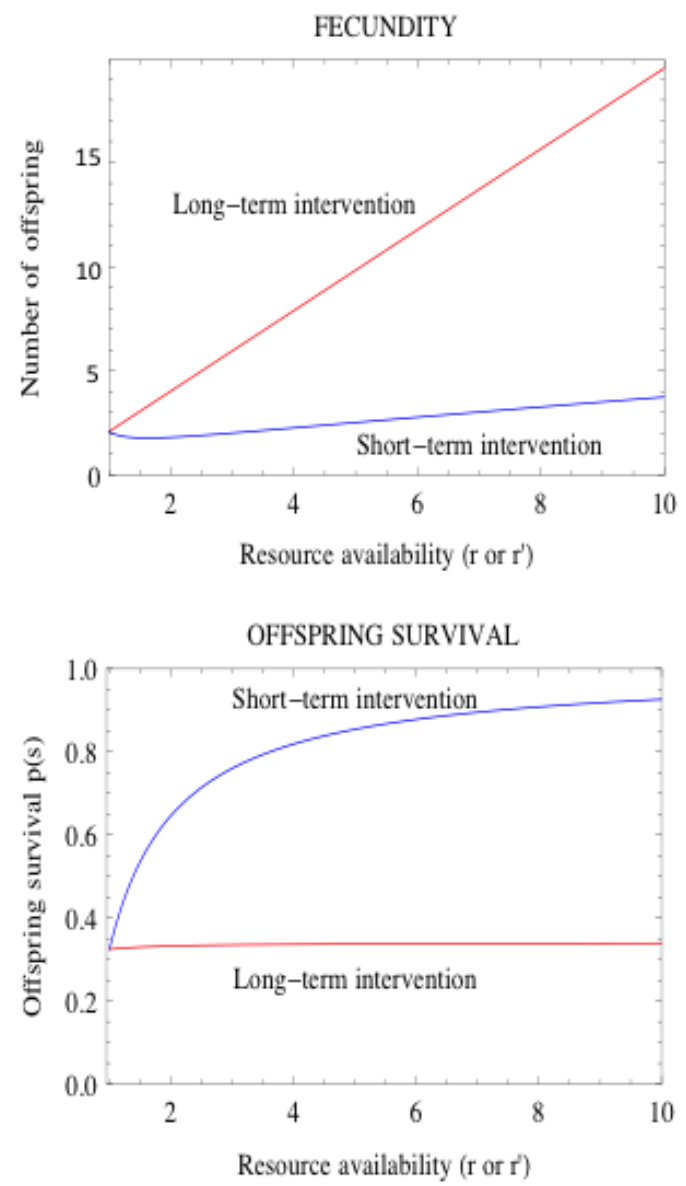